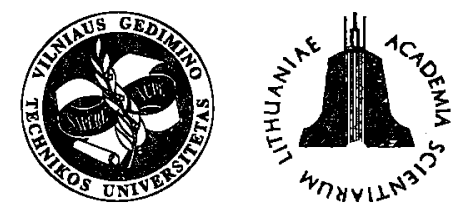

ISSN 1648-4142 TRANSPORT

http:/www.vtu.1t/english/editions

TRANSPORT - 2003, Vol XVIII, No 5, 193-197

\title{
IMPROVEMENT OF FREIGHT TRANSPORT TECHNOLOGIES AND IMPLEMENTATION OF NEW TECHNOLOGIES*
}

\author{
Adolfas Baublys \\ Transport Research Institute, Vilnius Gediminas Technical University, \\ Plytines g. 27, LT-2016VVilnius, Lithuania.E-mail: abaublys@takas.lt
}

Received 200303 25; accepted 20030901

\begin{abstract}
The synthesis of regional (urban) road transport freight system topological structure enables the assessment of impact exercised by various structural characteristics on the functioning of the system. For this reason the quality of project solutions in the stage of system structural synthesis increases.

Models of optimisation and simulation (included into the simulation procedure of optimisation of topological structure) have other possibilities as well. For instance, simulation model enables the solution of such various objectives of functional analysis of systems as, for example, the efficiency comparison analysis of stability of structure undergoing the changes of meanings of system parameters, the analysis of managing impacts of various types, loading technologies, etc.
\end{abstract}

Keywords: road transport freight, the simulation procedure of optimizing.

\section{Introduction}

Among other things, the technology of transportation involves the provision of services, i.e. freight haulage. Services embrace major operations (transpor-tation or haulage) and minor procedures (loading, un-loading, storage, control, documentation, etc.). The efficiency of technological operations may be defined in the terms of the following factors: consumption of energy and materials per ton $/ \mathrm{km}$ and quality and intensity of hauls. Moreover, technological operations of haulage should ensure safety of goods and be environmentally - friendly.

To improve the technology of transportation may seem as simple as to replace old transport facilities with the new ones. However, even the most advanced transport facilities may be not efficiently used. Therefore, the overall technological process of transportation should be improved. To achieve this numerous tests should be conducted, involving the determination of flows of goods, their constant control and forecasting, road network and traffic optimization, etc. The problems of improving freight transportation in long hauls and the carriage of 20-40 tons of goods were analysed in [1-5]. However, the analysis of carriage of goods of 50-1500 kg over small areas by transport facilities, say in the city, with the bottlenecks on the roads and ecological limitations in force,

*) Discussion of the results of the programme ,Transport: technologies, economic, environment, health. has not been practically made. In the present investigation some solutions to the above problems are offered.

\section{Major Solutions and Suggestions}

To determine the rational structure of the park of motor vehicles and the organizational form of transporttation the analysis of freight volume and the particular consignments in terms of time should be made. Since the carriage demand and consignment volume are random variables, their analysis in terms of time should be based on mathematical statistical data.

The structural analysis of the freight flows made allowed us to select and correct the methods of forecasting the flows of goods. It has been shown that forecasting based on mathematical expectation is the most appropriate. Statistical prediction based on a single point is less accurate.

In modelling transport networks and flows of traffic and goods a non - parametric approach to evaluating random factors seems to be more appropriate, because it has more advantages compared with the parametric approach. First, when the density of flows distribution is changing in time, the sufficiently accurate approximation is still practically feasible; second, this approach is not so error - sensitive in respect to the data used. After some time the statistical data on the flows of freight and vehicles should be updated and expanded. The larger the amount of information, the more expensive its storage, 
therefore, the required statistical evaluation should be made for the minimum amount of data. For this purpose mathematical models needed for the storage of statistical data have been developed and validated. The models were also developed for other cases, when the distribution density of random factors is determined in the following way:

a) by Bayas method;

b) by normal distribution;

c) by logarithmic normal distribution;

d) by non - logarithmic distribution;

e) by Veibool distribution.

Algorithms for setting the minimum amounts of data have been developed for the cases described above.

The synthesis of the regional (urban) system topological structure of freight road transport facilities allows us to assess the impact of various structural characteristics of the system on its performance. This leads to the increase of steps of synthesizing the structure of the design solution quality evaluation system.

Optimization and simulation models included into the simulation procedure of optimization may be used for other purposes as well. For example, the simulation model may be applied solving various problems of functional analysis of the system, such as the investigation of the stability of the structure with varying values of the system parameters as well as the comparative analysis of the impacts produced by major factors, loading technologies, etc.

The algorithm offered allows the operator to optimally determine the required average number of transport facilities according to the functioning capacities of the park of vehicles.

The methods of determining the optimal number of consignments and the way of transportation, when the total storage and carriage expenses are taken as an optimality criterion, are offered. An algorithm to optimize the road network connecting the stores has also been developed. In testing the algorithm it has been found that if the distribution density of the efficiency function is sufficiently high in the extremum zone, an independent random search is rather effective, even compared with the most of regular methods. First, the number of the variants considered is $200-500$. Second, a lot of the variants close to the global extremum can be identified, which, in most cases, may be interpreted as the uncertainty zone in further analysis of the incomplete initial data. Usually the above zone is determined by multicriteria optimization applying the regular methods.

A model of distribution of warehouses in the network of stores by optimizing transportation of goods has been developed. The efficiency function of the model consists of the following: fixed construction costs of large and small warehouses; variable costs of raw materials and carriage of goods from the warehouse to the store.
The conducted experimental research into the freight flows allows us to expand the theory of freight flows adding the following cases to which it may be applied:

a) in general, to a multiple freight flow;

b) to a multiple freight flow with one freight sender;

c) to maximizing the flow by minimizing the distance of carriage;

d) to multiple freight flows at minimum cost of transportation.

In theoretical analysis the relevant theorems have been proved with the actual examples provided.

Now let us consider in more detail the simulation procedure of optimization of the topological structure of a regional freight road transport system (KKTRS).

\section{Synthesis of the Topological Structure of Road Freight Transport Regional (Urban) System}

In designing a logistics center of transport distribution the topological structure of the area which is serviced by this centre should be worked out. In the above structure a number of the local warehouses, their capacity and location in the zone as well as the settlements (or the particular urban areas) which will be serviced by the particular warehouses, for instance, by those belonging to a commercial enterprise "MAXIMA", should be provided for.

For example, the network of the settlement (town) roads for freight transportation is expressed by the matrix $D=\left\|d_{i i^{\prime}}\right\|$, where $d_{i i^{\prime}}$ are the shortest routes in the road network between the $i$-th and the $i^{\prime}$-th points; $d_{i i^{\prime}}=d_{i^{\prime} i}$. The amount of goods to be carried in the particular region serviced by the particular company is also determined by the matrix $A^{K}=a_{i i^{\prime}}$, where $a_{i i^{\prime}}$ is the flow of goods from point $i$ to point $i^{\prime}$. The purpose of the topological synthesis of freight road transport system embracing a set of commercial enterprises in the region $E^{n}$, the route between the points $D$ and the flow of goods $A^{k}$, is to divide the settlements of a region into zones serviced by the warehouses $E_{j}\left(\bigcup_{j} E_{j}=E^{n}, E_{j} \cap E_{j^{\prime}}=\emptyset\right)$ and to determine the location of warehouses within zones $E_{t}$, minimizing the costs of goods transportation in the region. This is expressed in the following way:

$\sum_{j \in J} \sum_{i \in I i^{\prime} \in I} c_{1} d_{i i^{\prime}} x_{i j} x_{i^{\prime} j^{\prime}}+\sum_{j \in J} \sum_{j^{\prime} \in J} \sum_{k \in I} \sum_{k^{\prime} \in I} c_{2} d_{k k^{\prime}} y_{k j} y_{k^{\prime} j^{\prime}}+$

$\sum_{i \in I i^{\prime} \in I} \sum_{i i^{\prime}} x_{i j} x_{i^{\prime} j^{\prime}}+\sum_{j \in J} \sum_{j^{\prime} \in J} \sum_{k \in I} \sum_{k^{\prime} \in I_{j}} c_{1} d_{k k^{\prime}} y_{k^{\prime} j}+$

$\sum_{i \in I_{j}} \sum_{i^{\prime} \in I_{j}} a_{i i^{\prime}} x_{i j} x_{i j^{\prime}}$,

here, $x_{i j}=1$ if the point $i$ enters zone $j$ of the warehou- 
se; in the opposite case $x_{i j}=0 ; y_{i j}=1$, if the warehouse is located at the point $i$; for the opposite case $-y_{i j}=0$; $c_{1}$ - cost of goods transportation by low or medium functional capacity vehicles; $c_{2}$ - cost of transportation by high functional capacity lorries and trailers; $J$-a set of warehouse indices; $I_{j}-$ a set of indices of settlements in the $j$-th warehouse zone; $N$-a set of indices of the region settlements where warehouses may be located.

The first term in the formula (1) expresses the cost of goods transportation within a zone, while the 2 -nd term denotes the cost of goods transportation from one warehouse to another, and the $3-\mathrm{d}-$ the cost of goods carriage to and from the warehouse.

The synthesis of the topological structure of a freight road transport regional system (KKTRS) is performed solving two problems:

a) the distribution of a set of points supplying and receiving goods according to the zones serviced by the particular warehouses, minimizing the total freight flows disbalance between the warehouses;

b) determining the location of the warehouse within a zone, minimizing the total volume of work of delivering goods to a warehouse and taking them out of it. The total disbalance of cross - hauls will be the optimization criterion of the problem $a$, because this is the main cause why the functional capacity of vehicles and the hauls are not used to full advantage.

Let $P_{i}=\left\{E_{i / j}=1, \overline{2^{n}}\right\}$ be a family of all $E_{j}$ subsets of a region set of points $E^{P}=\{1,2, \ldots, i, \ldots, n\}$. Here, $n$ is the number of points in the region. In order to explain the $k$-th expansion of the set $E^{P}$, let us introduce the set $J_{k}$ which is such a set with the index $E_{j}$ that $\bigcup_{j \in J_{k}}=E^{P}, E_{j} \cap E_{j^{\prime}}=\emptyset, V_{j}, j^{\prime} \in J_{K}$. Then, the $k$ th expansion of the set $E^{P}$ is such a subfamily $P_{k}\left(P_{k} \subset R\right)$ that $P_{k}=\left\{E_{j} / j \in J_{k}\right\}$, where $J_{k}$ means a subset $E_{j}$ of the expansion. The disbalance of cross flows of goods of the warehouse of the zone serviced by the warehouse $j$ will be as follows:

$$
\alpha_{j}=\sum_{i \in E_{j}} \sum_{i^{\prime}=E_{j}}\left(a_{i i^{\prime}}-a_{i^{\prime} i}\right),
$$

here, $a_{i i^{\prime}}$ is the flow of goods from point $i$ to point $i^{\prime}$.

The total disbalance among the warehouses in the system for the $k$-th expansion is determined in the following way:

$$
z=\sum_{j \in J} \alpha_{j} x_{j}
$$

here, $x=\left\{\begin{array}{l}1, \text { if } j \in J_{k}, \\ 0, \text { if } j \notin J_{k},\end{array}\right.$

here, $J$ is a set of indices of the subset $E_{j}$, the potential zones serviced by warehouses.

In formalizing the problem of the system structure synthesis the requirements to strictural and functional KKTRS parameters are stated by the constraints of two types: 1) the constants of structural parameters of the system are commonly expressed by the analytical expression; 2) algorithmic expression is mostly used for functional parameters requirements (the sophisticated relationships of these parameters can not be expressed analytically).

The following analytically expressed constraints can be suggested:

The requirements that the subsets do not cross each other and cover the whole sets of the region points:

$$
\sum_{j \in J} t_{i j} x_{j}=1, \quad i \in E^{P},
$$

here, $t_{i j}=\left\{\begin{array}{l}1, \text { if } i \in E_{j}, \\ 0, \text { if } i \notin E_{j} .\end{array}\right.$

The number of the region warehouses serviced is:

$$
Q_{\min }^{t} \leq \sum_{j \in J} x_{j} \leq Q_{\max }^{t},
$$

here, $Q_{\min }^{t}, Q_{\max }^{t}-$ permissible and maximum numbers of warehouse zones in the system for the warehouse number in each zone:

$$
\sum_{i \in E^{P}} b_{i} t_{i j} \geq 1, \quad j \in J,
$$

here, $b_{i}=\left\{\begin{array}{l}1, \text { if a warehouse may be set up at point } i, \\ 0 \text {-for the opposite case; }\end{array}\right.$

$$
G_{\min }^{l} \leq \sum_{i \in E^{P}} t_{i j} \leq G_{\max }^{l}, j \in J,
$$

here, $G_{\min }^{l}, G_{\max }^{l}-$ permissible minimum and maximum numbers of points in a warehouse zone:

$$
d_{i i^{\prime}} t_{i j} t_{i^{\prime} j} \leq L^{l}, \forall i, i^{\prime} \in E^{P}, j \in J,
$$

for the length of the route between warehouse $j$ and the points of its zone:

$$
d_{i i^{\prime}} \leq L_{T}^{l}, \forall i^{\prime}: b_{i^{\prime} j}=1, \quad j \in J, \quad i \in E^{P},
$$

here, $L_{T}^{l}$ is the permissible length of route between the warehouse $j$ and the points of its zone; the total volume of goods in a flow handled in warehouse $j$ : 


$$
\sum_{i \in E^{P}} \sum_{i \in E^{P}}\left(a_{i i^{\prime}}+a_{i^{\prime} i}\right) t_{i j}\left(1-t_{i j}\right) \leq \hat{P}_{j}, \quad j \in J,
$$

here, $\quad \hat{P}_{j}$ - the capacity of the terminal $j$ allowing to handle goods in the period of time $\tau_{m}$.

\section{The Simulation Procedure of Optimizing the To- pological Structure of Freight Road Transport Re- gional System (KKTRS)}

In general the simulation procedure of optimizing KKTRS topological structure consists of the following major steps:

1) generation of the particular variant of the structure;

2) checking of the compliance of the variant with the stated analytical constraints;

3) computer - aided experiment with the simulation model of the structural variant with permissible analytical constraints.

The optimization algorithm of the simulation procedure consists of two optimization models.

A special algorithm generates the initial family of the subset $R_{D}=\left\{E_{j} / E_{i}, j \in J\right\}$ and selects all $E_{j} \in R$. Then, the algorithm is implemented in the following blocks:

Block 1 . The initiation of the simulation procedure of optimization. Let $S_{j}=\left\{i l_{t j}=1\right\}$ be a set of the separate elements of the $j$-th column indices of $\mathrm{T}$ matrix; $R_{i}=\left\{j t_{i j}=1\right\}$ be a set of the separate elements of the $i$-th row of $\mathrm{T}$ matrix; $W=\left\{j x_{j}=1, v_{j}: t_{i j}=1 \rightarrow W=\sum_{j \in W} t_{i j}=1\right\}-$ a set of the indices of variables of a partial solution; $S_{w}+\left\{i / \sum_{j=w} t_{i j}=1\right\}=\bigcup_{j \in w} S_{j}$ - a lot point; $V=Q-S_{w}$; $X_{m}=\left\{S_{j}, x_{j}=1\right\}-$ a set of points of solution of problem $a$ (the $m$-th variant of the division of the points serviced by a warehouse into zones); $V=Q-S_{w} ; Y_{m}=\left\{i / y_{i j}=1\right\}-$ optimal solution of the problem $b$ of the variant $X_{m}$ expansion; $\bar{X}_{m}=\left\{X_{m} Y_{m}\right\}$ - the $m$-th variant of the system topological structure; $x=\left\{X_{m}\right\}-$ a family of the obtained variants of the topological structure; $H$ - the current value of the efficiency function maximum; $F(w)=\sum_{j \in w} a_{j}$ - the current value of partial solution of the efficiency function; $F\left(X_{m}\right)$ - the value of the complete solution of the efficiency function. First, $W=S_{w}=\varnothing, v=Q, X=\varnothing$ are determined. Then, the 2-nd block is handled.

The 2 -nd block. Generation of the family $R_{D}$. The matrix $T=l_{i j}$ is generated, with every $j$-th column corresponding to the set $E_{j} \in E$ and being $n$ the componential cortege $\left\langle l_{i j}, \ldots, l_{n j}\right\rangle$ above the set $\{0,1\}$. Then all lexicographically arranged $i$ componential corteges $(i=1, n)$ satisfying the specified constraints are generated. All the corteges are checked satisfying the constraints. If so, the value $\alpha_{j}$ of the cortege is calculated and included into the matrix T. In the opposite case, another cortege is generated. Then we go on to the 3-d block.

The 3-d block. The matrix B is transformed. The columns $S_{j}$ are included into the lists $L_{i} . S_{j}$ is included into the list $L_{i}$ then and only then, when $t_{1 j}=t_{2 j}=\ldots=t_{(i-1) j}=0$, while $t_{i j}=1 . S_{j}$ of each $j$ th list is transformed according to $\alpha_{j}$.Thus $n$ lists are formed, while every set $S_{j}$ is included in only one list and some lists may be blank. Then, we go on to the 4-th block.

The 4-th block. It is aimed to make a list $L_{i^{*}}$ and column $S_{j^{*}} \in L_{i^{*}}$. The number of the list is chosen as $i^{*}=\min \{i i \in v\}$. The column $S_{j^{*}}$, which $\min \left\{\alpha_{j} j \in L_{i^{*}}\right\}$ corresponds, is chosen from the list $L_{i^{*}}$. Then, we may go on to the 5-th block.

The 5-th block. A variable to be introduced into the partial solution is determined. In the list $L_{i *} \alpha_{j}$, such $S_{n} \in L_{i *}$ is sought (beginning with $S_{j^{*}}$ in an ascending order) that $S_{w} \cap S_{n}=\varnothing$ and $F(w)+\alpha_{j} \leq H$. If such $S_{n}$ exists, the 7-th block is considered, if not, the 6-th block is handled.

The 6-th block. If $w=\varnothing$, we may go on to the 14th block. If $w \neq \varnothing$ and $S_{k}$ is the last column in $S_{w}$, then $k$ is taken out of $W$ and $S_{k}$ out of $S_{w}$. As a result the following changes take place: $F(w): w=w\{k\}$, $F(w)=F(w)-\alpha_{k}$ and $S_{w}=S_{w} /\left\{i / i \in S_{k}\right\}$. Let $i^{*}$ be an index of the list containing $S_{k}$. Then we go on to the 5-th block.

The 7-th block. A new variable $x_{n}$ is introduced into the partial solution $w=w \cup\{n\}$, $S_{w}=S_{w} \cup\left\{i / i \in S_{n}\right\}, F(w)=F(w)+\alpha_{n}$. If $S_{w} \neq Q$, we may go on to the 4-th block. If $S_{w}=Q$, the 8-th block is handled.

The 8-th block. A new expansion variant $\left\{w, S_{\bar{w}}\right\}$ should be replaced. Then $H=F(w)$, $\bar{X}_{m}=\left\{S_{j} / j \in w\right\}$, we may go on to the 9-th block

The 9-th block. The optimal arrangement of $Y_{m}$ variant for the obtained $X_{m}$ expansion version is determined. In this way, the variant $X_{m}$ of the topological structure KKTRS, permissible within analytical constraints $X_{m}=\sigma$, is formed. Then, we go on to the 10-th block.

The 10-th block. The initiation of simulation. Changing of the values of structural parameters of simulation according the structural variant $X_{m}$, we go on to the 11-th block. 
The 11-th block. Simulation. The values $k_{k}, k_{r}$, $T_{m}, \tau_{m j}$ and $\nu_{t}$ of the structural variant $\bar{X}_{m}$ are obtained. Then we go on to the 12-th block.

The 12-th block. The variant $\bar{X}_{m}$ of the structure may be tested as to its correspondence with algorithmically determined constraints. If $\bar{X}_{n} \in \overline{\bar{\sigma}}$, then we go on to the 13-th block. If $X_{n} \notin \bar{\sigma}$, we go on to the 4-th block.

The 13-th block. The variant of the structure $X_{m}: X=X \cup\left\{X_{m}\right\}$ permissible according to all constraints is fixed and the maximum $H=F\left(\bar{X}_{m}\right)$ is changed. Then, we go on to the 5-th block.

The 14-th block. Printing of the results. If $X=\varnothing$, then the suitable variants of the topological structure KKTRS have not been found. In the opposite case the variants of the structure are such that $F\left(\bar{X}_{\text {opt }}\right)=H$. The work with the algorithm is over.

Optimization and simulation models used in the procedure may have more applications. For example, the simulation model allows us to solve various problems of functional analysis of the systems, such as the study of the stability of the structure under the condition of varying system parameters, the investigation of the effects of the most important factors, the analysis of the efficiency of various loading technologies, etc.

\section{Conclusions}

1. In big cities (Vilnius, Kaunas, etc.), large chains of stores should constantly study the flows of goods belonging to them as well as the flows of transport facilities delivering these goods to trade outlets and warehouses. Institutions of local government should control them and stimulate their interest in the rational solution of these problems.

2. The algorithm for the optimisation of the local road network among the trade points is created. During the course of algorithm testing it was proved that if the purpose function meanings distribution density in an extremum zone is sufficiently large, the independent random search is efficient enough even in comparison with regular methods. Firstly, there are 200-500 analysed variants. Secondly, it is found out how many variants (close to the global extremum), which in many cases may be interpreted as the zone of indeterminability, later assessed incomprehensive initial information. Usually such a zone is found by the way of multicritical optimisation with the application of regular methods.

3. The model of dislocation of warehouses in the trade network is created in the way of optimisation of transportation of goods. In the model the purpose function consists of: fixed costs of building large and small warehouses; the variable costs of stock and goods transportation between warehouses and trade points.

4. The performed experimental research into freight flows supplements the theory with the following cases:

a) for flows of most freight in general;

b) for multiple freight flow with a common freight consignor;

c) maximisation of the flow by the minimisation of its transportation distance;

d) for flows of most freights by the least costly transportation.

In the course of theoretical research corresponding theorems were proved, the research basing on concrete practical examples.

\section{References}

1. Guelat, J.; Florian, M.; Crainic, T.-G. A Multimode Multiproduct Network Assignment Model for Strategic Planning of Freight Flows. Transportation Science, Vol 24, No 1. February, 1990, p. 25-39.

2. Zhan, F.-B.; Noon, Ch.-E. Shortest Path Algorithms: An Evaluation using Real Road Networks. Transportation Science, Vol 32, No 1. February, 1998, p. 65-73.

3. Nuzzolo, A.; Russo, F.; Crisali, U. A Doubly Dynamic Schedule - based Assignment Model for Transit Network. Transportation Science, Vol 35, No 3. August, 2001, p. 268 285.

4. Bodin, L.; Mingozzi, A.; Baldacci, R.; Ball, M. The Roll on - Roll off Vehicle Routing Problem. Transportation Science, Vol 34, No 3, August, 2000, p. 256-271.

5. Baublys, A. Assessment of statistical probability of the technological transportation process. Transport, Vol XVII, No 4, 2002, Vilnius: Technika, p. 147-151. 